\title{
Effects of single therapeutic doses of promethazine, fexofenadine and olopatadine on psychomotor function and histamine-induced wheal- and flare-responses: a randomized double-blind, placebo-controlled study in healthy volunteers
}

\author{
Hiroyuki Kamei · Ami Isaji $\cdot$ Yukihiro Noda \\ Kazuhiro Ishikawa $\cdot$ Koji Senzaki · Kiyofumi Yamada • \\ Kazumitsu Sugiura · Yasushi Tomita · Toshitaka Nabeshima
}

Received: 8 January 2011/Revised: 9 November 2011/Accepted: 14 November 2011/Published online: 1 December 2011

(C) The Author(s) 2011. This article is published with open access at Springerlink.com

\begin{abstract}
Since most first-generation antihistamines have undesirable sedative effects on the central nervous systems (CNS), newer (second-generation) antihistamines have been developed to improve patients' quality of life. However, there are few reports that directly compare the antihistaminic efficacy and impairment of psychomotor functions. We designed a double-blind, placebo controlled, crossover study to concurrently compare the clinical effectiveness of promethazine, a first-generation antihistamine, and fexofenadine and olopatadine, second-generation antihistamines, by measuring their potency as peripheral inhibitors of histamine-induced wheal and flare. Further, we investigated their sedative effects on the CNS using a battery of psychomotor tests. When single therapeutic doses of fexofenadine $(60 \mathrm{mg})$, olopatadine $(5 \mathrm{mg})$ and promethazine $(25 \mathrm{mg}$ ) were given in a double-blind manner to 24 healthy volunteers, all antihistamines produced a
\end{abstract}

H. Kamei · A. Isaji

Department of Clinical Pharmacy Practice and Health Care

Management, Faculty of Pharmacy, Meijo University,

Nagoya 468-8503, Japan

Y. Noda

Division of Clinical Sciences and Neuropsychopharmacology, Graduate School of Pharmaceutical Sciences, Meijo University,

Nagoya 468-8503, Japan

Y. Noda $\cdot$ K. Yamada $\cdot$ T. Nabeshima

Japanese Drug Organization of Appropriate

Use and Research, Nagoya 468-8503, Japan

K. Ishikawa $\cdot$ K. Senzaki $\cdot$ K. Yamada

Department of Neuropsychopharmacology and Hospital

Pharmacy, Nagoya University Graduate School of Medicine,

Nagoya 466-8550, Japan significant reduction in the wheal and flare responses induced by histamine. In the comparison among antihistamines, olopatadine showed a rapid inhibitory effect compared with fexofenadine and promethazine, and had a potent effect compared with promethazine. In a battery of psychomotor assessments using critical flicker fusion, choice reaction time, compensatory tracking, rapid visual information processing and a line analogue rating scale as a subjective assessment of sedation, promethazine significantly impaired psychomotor function. Fexofenadine and olopatadine had no significant effect in any of the psychomotor tests. Promethazine, fexofenadine and olopatadine did not affect behavioral activity, as measured by wrist actigraphy. These results suggest that olopatadine at a therapeutic dose has greater antihistaminergic activity than promethazine, and olopatadine and fexofenadine did not cause cognitive or psychomotor impairment. 
Keywords Antihistamines - Psychomotor performance . Wheal and flare $\cdot$ Fexofenadine $\cdot$ Olopatadine

\section{Introduction}

Antagonists of histamine $\mathrm{H}_{1}$ receptors, antihistamines have been widely used for the treatment of seasonal and perennial allergic rhinitis and chronic idiopathic urticaria. Classical (first-generation) antihistamines such as $d$-chlorpheniramine, diphenhydramine and promethazine, however, have undesirable side effects including sedation, at therapeutic doses $[8,27,30]$. Sedation induced by antihistamines impairs cognitive and psychomotor functions $[13,24,39]$. Notably, daytime sedation disturbs the ability to work, and excessive sedation reduces the patient's compliance with treatment regimens [25].

The sedative effect of antihistamines on the central nervous system (CNS) is due to their ability to cross the blood-brain barrier and to block histamine neurotransmission through central histamine $\mathrm{H}_{1}$ receptors. In fact, first-generation antihistamines have been shown to occupy a large proportion of histamine $\mathrm{H}_{1}$ receptors in the human brain by positron emission tomography (PET) [41]. A number of newer (second-generation) antihistamines, which do not readily cross the blood-brain barrier, have been developed to relieve side effects on the CNS and to improve quality of life $[22,32,36]$. The second-generation antihistamines fexofenadine and olopatadine, which have potent antihistaminic properties and fewer CNS side effects, are now widely used to treat seasonal allergic rhinitis in Japan. Fexofenadine and olopatadine have been approved in Japan for the treatment of allergic disorders at a recommended total daily dose of $120 \mathrm{mg}(60 \mathrm{mg}$ twice daily) and $10 \mathrm{mg}$ (5 mg twice daily), respectively. In healthy volunteers, fexofenadine did not have any disruptive effects on psychomotor or cognitive performance, when administered at doses of up to $360 \mathrm{mg}$ [9, 10, 14]. On the other hand, olopatadine at $10 \mathrm{mg}$, which is double the standard oral dose, caused a decrease in behavioral activity as measured by wrist actigraphy, although it did not affect psychomotor/cognitive performance [14]. However, further comparative studies should be carried out to determine the clinical profile of antihistamines in terms of the relationship between their efficacy (antihistaminic activity; benefit) and psychomotor functions (risk) using the therapeutic doses. The efficacy of antihistamines has been evaluated with histamine challenge tests using iontophoresis [29]. Iontophoresis is a non-invasive method of histamine application to induce an inflammatory skin response (wheal/flare), which is useful to compare the effects of antihistamines.
Thus, this study was designed to investigate the effects of fexofenadine, olopatadine and promethazine, a positive control, at the single therapeutic doses on psychomotor/ cognitive function and on histamine-induced cutaneous responses in healthy Japanese.

\section{Materials and methods}

\section{Subjects}

The present study was approved by the institutional review board of the Medical School Hospital of Nagoya University (an approval number: 208008).

All subjects were given written informed consent with respect to their participation in the study. Twenty-four healthy Japanese volunteers (twelve females, twelve males) aged between 18 and 22 (mean \pm SE: $19.3 \pm 0.3$ ) years were entered into the study. None of the subjects had evidence of previous or current physical and mental illness, including allergies, on the basis of medical history, a clinical examination, a 12-lead electrocardiogram, and standard laboratory tests of plasma and urine. None had a history of alcohol or drug abuse or drug allergy. Subjects received no medication for 2 weeks before and during the study.

Study design and treatments

The study was a randomized, double-blind, placebocontrolled, crossover study with four periods of treatment each separated by a washout period of 7 days. During each period, subjects received a single therapeutic dose of each of the study drugs: promethazine $(25 \mathrm{mg})$, a first-generation antihistamine, and fexofenadine $(60 \mathrm{mg})$ and olopatadine $(5 \mathrm{mg})$, second-generation antihistamines, and placebo. The study drugs were assigned according to the randomization list. All treatments were supplied in capsules, which were identically matched in size, color and shape to respect the double-blind nature of the study. The study drugs were administered with about $150 \mathrm{ml}$ of water. The Pharmacokinetic parameters of promethazine $(25 \mathrm{mg}$ ) [20], fexofenadine $(60 \mathrm{mg})$ [33] and olopatadine $(5 \mathrm{mg})$ [5] are as follows: $T(\max )$ of them are $2.7,2.2$ and $1.0 \mathrm{~h}, T(1 / 2)$ of them are $12.7,9.6$ and $8.8 \mathrm{~h}$, respectively.

\section{Procedures}

The examination was performed over 4 weeks at intervals of 1 week. Subjects attended the examination site on the day before each of the tests, where breath alcohol, health and medication were checked. The day before the first test, 
Fig. 1 Study schedule

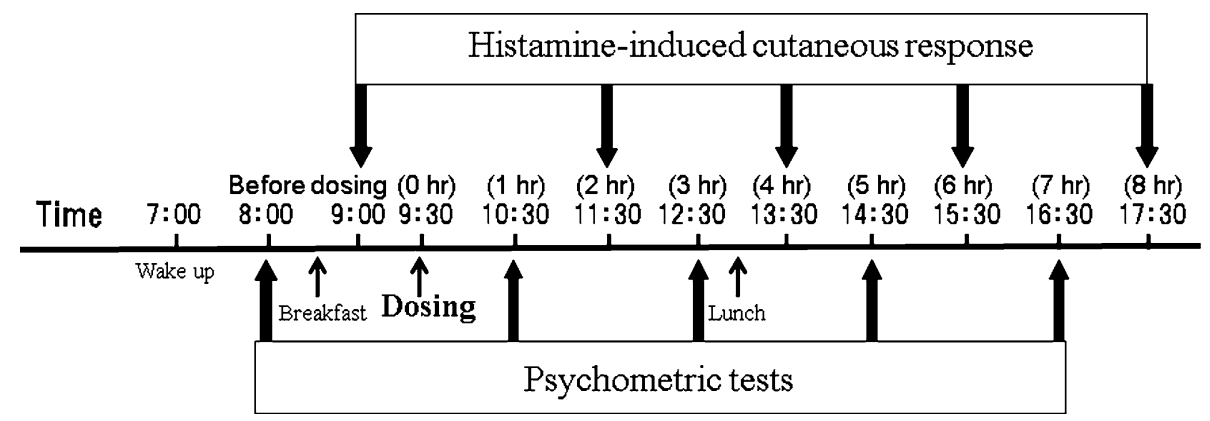

they received sufficient training (at least three times) for the psychometric tests in order to eliminate the effects of learning [23]. At each visit, they were instructed to go to bed in a single room at a hotel near the examination site (Nagoya University Hospital) at 2300 hours.

The study schedule is shown in Fig. 1. On each of the test days, subjects were awakened at 0700 hours, and moved to the examination room. An alcohol breath test was first conducted. Two baseline measurements were undertaken before medication. First, at 0800 hours, subjects undertook a psychometric test. Second, at 0900 hours, subjects were assessed for a histamineinduced cutaneous response. Breakfast was taken between 0830 and 0900 hours. Study drugs were administered at 0930 hours. Then, the psychometric tests were carried out $1,3,5$ and $7 \mathrm{~h}$ after the drug administration, and the histamine-induced cutaneous response was examined 2, 4, 6 and $8 \mathrm{~h}$ after the drug administration. The duration of each test in the psychometric test battery was for $\sim 30 \mathrm{~min}$.

The consumption of alcohol, nicotine, caffeine and grapefruit was prohibited for 2 days before and during testing. Food consumption was strictly controlled the night before and during testing. Adverse events and concomitant medications were recorded at each visit.

\section{Assessments}

Histamine-induced cutaneous response

Histamine iontophoresis-induced wheal- and flare-responses were measured, according to the method of Takahashi et al. [33]. Histamine dihydrochloride was dissolved in distilled water at a concentration of $10 \mathrm{mg} / \mathrm{ml}$. The histamine solution was applied to the participant's forearm (alternating between the right and left sides) by an iontophoreser (UI-2060, BS Medical, Tokyo, Japan), with cotton wool. A constant current of $0.1 \mathrm{~mA}$ was applied for $1 \mathrm{~min}$. The areas of histamine-induced wheal- and flare-response were assessed $15 \mathrm{~min}$ after the application via a planimetric evaluation, which was performed using NIH imaging computer software. The sizes of wheal- and flare- areas were expressed as a percentage of the baseline histamine response before the intake of the study drug.

\section{Psychometric tests}

Critical flicker fusion

Critical flicker fusion (CFF) was used as a means of measuring overall CNS arousal using the ability to discriminate discrete 'bits' of sensory information [7].The test device was composed of four light-emitting diodes arranged in a $1-\mathrm{cm}$ square. The diodes were held in foveal fixation $1 \mathrm{~m}$ from the subject. The lights were flicked on and off at a constantly increasing or decreasing rate. Subjects were required to discriminate flicker from fusion, and vice versa. Individual thresholds were determined as the mean of each threshold in four ascending (flicker to fusion) and four descending (fusion to flicker) measurements.

\section{Choice reaction time}

The choice reaction time (CRT) was used as a sensitive measure of drug-induced changes in sensorimotor performance [6]. The test device was composed of a central starting button, six red buttons aligned in the shape of a fan, which were equally separated from the starting button, and corresponding response buttons located in front of each red light, and six green lights located behind each red light. Subjects placed the index finger of their preferred hand on the starting button and then were required to extinguish one of six equidistant red lights, illuminated at random, by touching the corresponding response button in front of the light as quickly as possible. The green light was used as notice indicator to let the subject know which of the red lights to focus on. The time between the red light coming on and the finger being released from the starting button was taken as a recognition reaction time of CRT. The mean reaction time for 48 stimulus presentations was recorded. 
Compensatory tracking test

The compensatory tracking test (CTT) was used as a means to assess divided attention [11]. Subjects were required to keep a cursor in alignment with a moving target on a visual display unit screen using a mouse. The evaluation measure of this tracking task was the mean difference between the centers of the target and cursor in pixels, sampled 5 times per second, during the 9-min test period. Lower scores are indicative of more accurate tracking.

In addition, a peripheral awareness task is included in which the subject responds to a stimulus presented in the periphery of vision, while simultaneously attending to the tracking task described above. One hundred stimuli appear at different location, which randomly appear at four corners on the screen. Stimuli duration is $3 \mathrm{~s}$, and stimuli interval is 3-6 s. Subjects should control the mouse to track the target, and simultaneously click the left side of mouse as soon as possible if they notice the stimuli at a corner on the screen. The mean reaction time to these stimuli over the trial period was taken as the response measure for this component of the divided attention task.

Rapid visual information processing

Rapid visual information processing (RVIP) was used as a means to assess attention performance [40]. Subjects were required to monitor a series of single digits (0-9) appearing on the screen at a rate of 100 digits every minute and respond to consecutive sequences of three odd or even digits using a mouse button during the 9-min test period. The evaluation measures are the number of correct responses. The RVIP task was performed only at baseline and $3 \mathrm{~h}$ after the administration of study drugs. This task was followed by the CFF, the CRT and the CTT at baseline and $3 \mathrm{~h}$ after the administration of study drugs. Because this task requires high concentration for $9 \mathrm{~min}$ and is mentally taxing to subjects, the RVIP task is done only twice a day.

Line analogue rating scale

The line analogue rating scale (LARS) was employed as a measure of the subjective effects of psychoactive drugs [6]. Subjects were required to mark a point, which represented how they felt, on 100-mm line analogue scales. Subjects should mark degree of feeling tiredness, drowsiness and alertness using this scale which was defined that values 0 showed that they feel no tiredness, no drowsiness, and are not alert, value 100 showed that they feel very tired, very drowsy, and are very alert. The mean score of the rating of 'tiredness', 'drowsiness' and 'not alert' was taken as a measurement of sedation [10].
Wrist actigraphy

Actigraphy has been shown to be capable of measuring reductions in behavioral activity (sedation) caused by psychoactive drugs [31]. On each test day, a watch-type wrist actigraph (Actiwatch, Cambridge Neurotechnology Ltd, UK) was placed on the wrist of subjects to detect threedimensinal movements, and the behavioral activity of subjects was measured from 1-h pre-dose to 7-h post-dose. The wrist actigraph contained a piezoelectric transducer that detects motion in all three axes and generates a signal voltage. In the zero crossing mode, each crossing of the reference voltage during an epoch is counted, which gives a measure of the frequency but not the intensity (amplitude) of the movements. Mean behavioral activity over the entire recording period was automatically calculated for \% sleeplike behavior using ACTION3 software and its validated sleep/wake algorithm (Ambulatory Monitoring Inc, USA).

Statistical analysis

The data were analyzed using the one-way ANOVA or two-factor factorial ANOVA, followed by Tukey-Kramer test, regarding changes from baseline measurements. The one-way ANOVA was used for the RVIP test, and twofactor factorial ANOVA was used for the histamineinduced cutaneous response (wheals and flare) and the psychomotor tests such as CFF, CRT, CTT, actigraphy, and LARS. The factors of two-factor factorial ANOVA were treatment (4 levels: placebo, promethazine, fexofenadine and olopatadine) and time (4 levels: histamine-induced cutaneous response; 2, 4, 6 and $8 \mathrm{~h}$, psychometric tests; 1 , 3,5 and $7 \mathrm{~h}$ ). In the post hoc pairwise comparisons between the treatment means, Tukey-Kramer test was used for the histamine-induced cutaneous response (wheals and flare) and the psychomotor tests such as CFF, CRT, CTT, RVIP, and LARS. In order to ensure that there were no significant differences across tasks and conditions at pretreatment, baseline statistical analyses were conducted and used for comparison to post-treatment results. A $P$ value of $<0.05$ was defined as statistically significant.

\section{Results}

Histamine-induced cutaneous response

All 24 subjects completed the four periods of the study.

Histamine iontophoresis produced a marked wheal- and flare-response accompanied by itching. There was no difference in baseline data for the wheal and flare components of the histamine-induced cutaneous response among the treatment groups. 
The effects of antihistamines on wheals induced by the histamine application are shown in Fig. 2a. The two-factor ANOVA showed a significant main effect for treatment $[F(3,276)=12.36, P<0.01]$, time $[F(3,276)=8.06$, $P<0.01]$ and interaction between treatment and time $[F(9,276)=1.96, P<0.05]$. Post hoc pair-wise comparisons showed that fexofenadine $(P<0.01)$ and olopatadine $(P<0.01)$ overall had a significantly greater inhibitory effect on wheals than did the placebo. Analysis of the time course showed significant inhibition with promethazine in comparison to placebo, only at 4-h post-dose. The inhibitory effect of fexofenadine was significantly different from that of placebo at 4- and 8-h post-dose. Olopatadine had a significant effect on wheals in comparison to placebo and promethazine at 2-, 4-, 6- and 8-h postdose. Promethazine, fexofenadine and olopatadine caused maximum inhibition at $4 \mathrm{~h}$, i.e., 52, 80 and $99 \%$ of the baseline level, respectively. The marked inhibition of wheals by olopatadine continued during 4 and $8 \mathrm{~h}$. The area under the effect curve (AUC) for changes from baseline was used to assess the overall effect of the treatment. AUC from baseline to the last assessment was calculated using the trapezoidal rule. As shown in the upper graph in Fig. 2a, a one-way ANOVA of the effects of antihistamines on wheals induced by the histamine application, showed a significant main effect for treatment $[F(3,92)=13.03, P<0.01]$. Post hoc pair-wise comparisons showed that fexofenadine $(P<0.05)$ and olopatadine $(P<0.05)$ had a significantly greater inhibitory effect on wheals than the placebo. The inhibitory effect of olopatadine $(P<0.05)$ was significantly different from that of promethazine. There was no significant difference between olopatadine and fexofenadine in their inhibitory effects on wheals.

With regard to inhibition of the flaring reaction (Fig. 2b), a two-factor ANOVA showed a significant main effect for treatment $[F(3,276)=7.79, P<0.01]$ and time $[F(3,276)=6.80, P<0.01]$, although the effect of interaction between treatment and time was not significant $[F(9,276)=0.65, P=0.75]$. Post hoc pair-wise comparisons showed that fexofenadine $(P<0.01)$ and olopatadine $(P<0.01)$ overall had a significantly greater inhibitory effect on flares than the placebo. Analysis of the time course showed a significant effect with promethazine in comparison to placebo, only at 4 -h post-dose. The inhibitory effects of fexofenadine were significantly different from those of the placebo at 4,6 and $8 \mathrm{~h}$. The inhibitory effects of olopatadine were significantly different from those of the placebo at 2, 4, 6 and $8 \mathrm{~h}$, and from promethazine at $4 \mathrm{~h}$. The inhibitory effect of each drug on flares was similar to that on wheals. With analysis of AUC for changes from baseline, a one-way ANOVA of the effects of antihistamines on flares showed a significant

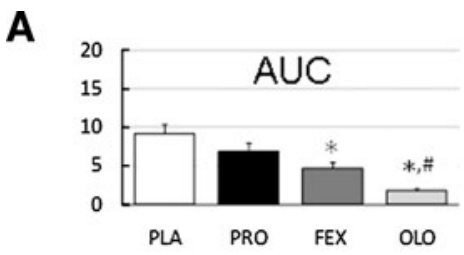

PLA: Placebo

PRO : Promethazine

FEX: Fexofenadine

OLO : Olopatadine
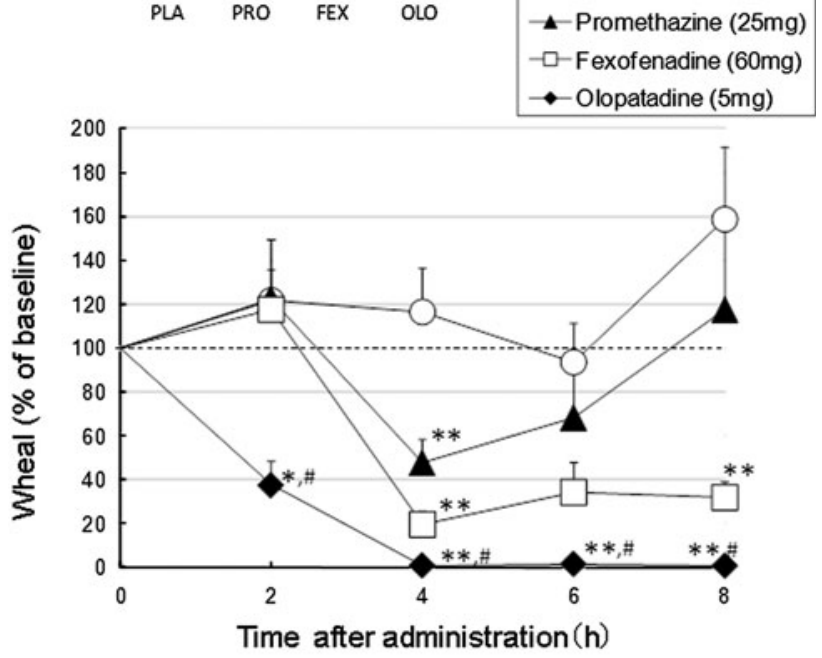

B

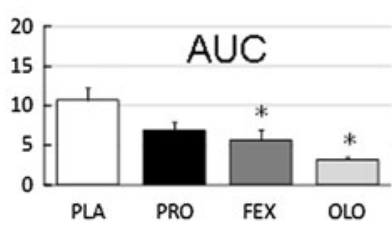

PLA: Placebo

PRO : Promethazine

FEX: Fexofenadine

OLO : Olopatadine
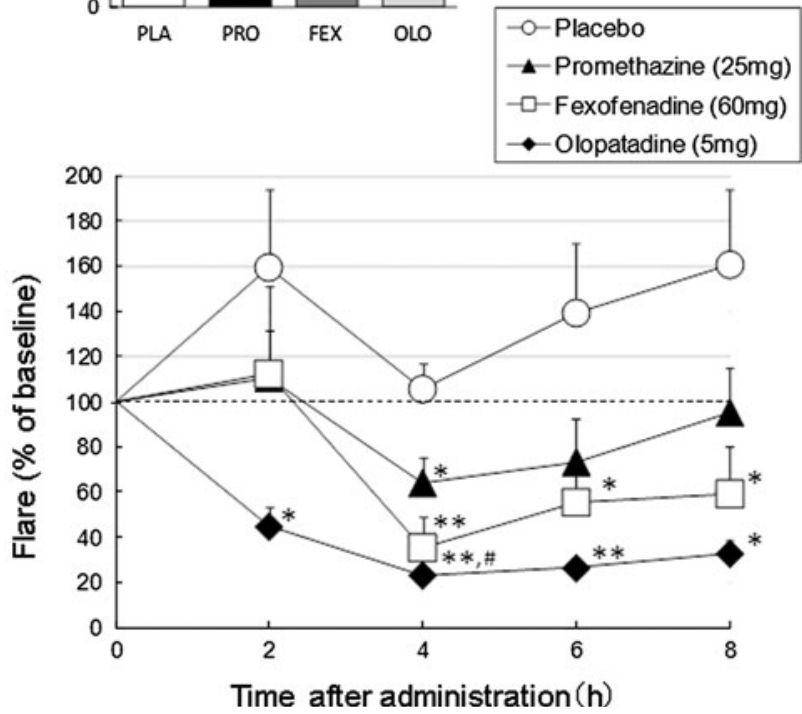

Fig. 2 Effects of antihistamines on histamine-induced wheal (a) and flare (b) responses. Each value is expressed as a percentage of the baseline, and is the mean \pm SEM for 24 subjects. Each upper graph in $\mathbf{a}$ and $\mathbf{b}$ shows the corresponding area under the effect curve (AUC) for changes from baseline of wheal (a) and flare (b) responses. ${ }^{*} P<0.05,{ }^{* *} P<0.01$ compared with placebo, ${ }^{\#} P<0.05$ compared with promethazine

main effect for treatment $[F(3,92)=7.69, P<0.01]$, as shown in the upper graph in Fig. 2b. Post hoc pair-wise comparisons showed that fexofenadine $(P<0.05)$ and 
olopatadine $(P<0.05)$ had significantly greater inhibitory effects on flares than the placebo. There was no significant difference between olopatadine and fexofenadine in their inhibitory effects on flares.

Assessment of cognitive and psychomotor function

There was no difference in baseline data for each psychometric parameter among the treatment groups. The effects of antihistamines on CFF, CRT, and CTT are shown in Fig. 3. The effects of antihistamines on RVIP, LARS and wrist actigraphy are shown in Fig. 4.

For the CFF test (Fig. 3a), a two-factor ANOVA showed a significant main effect of treatment $[F(3,276)=5.62$, $P<0.01$ ], although the effect of time was not significant
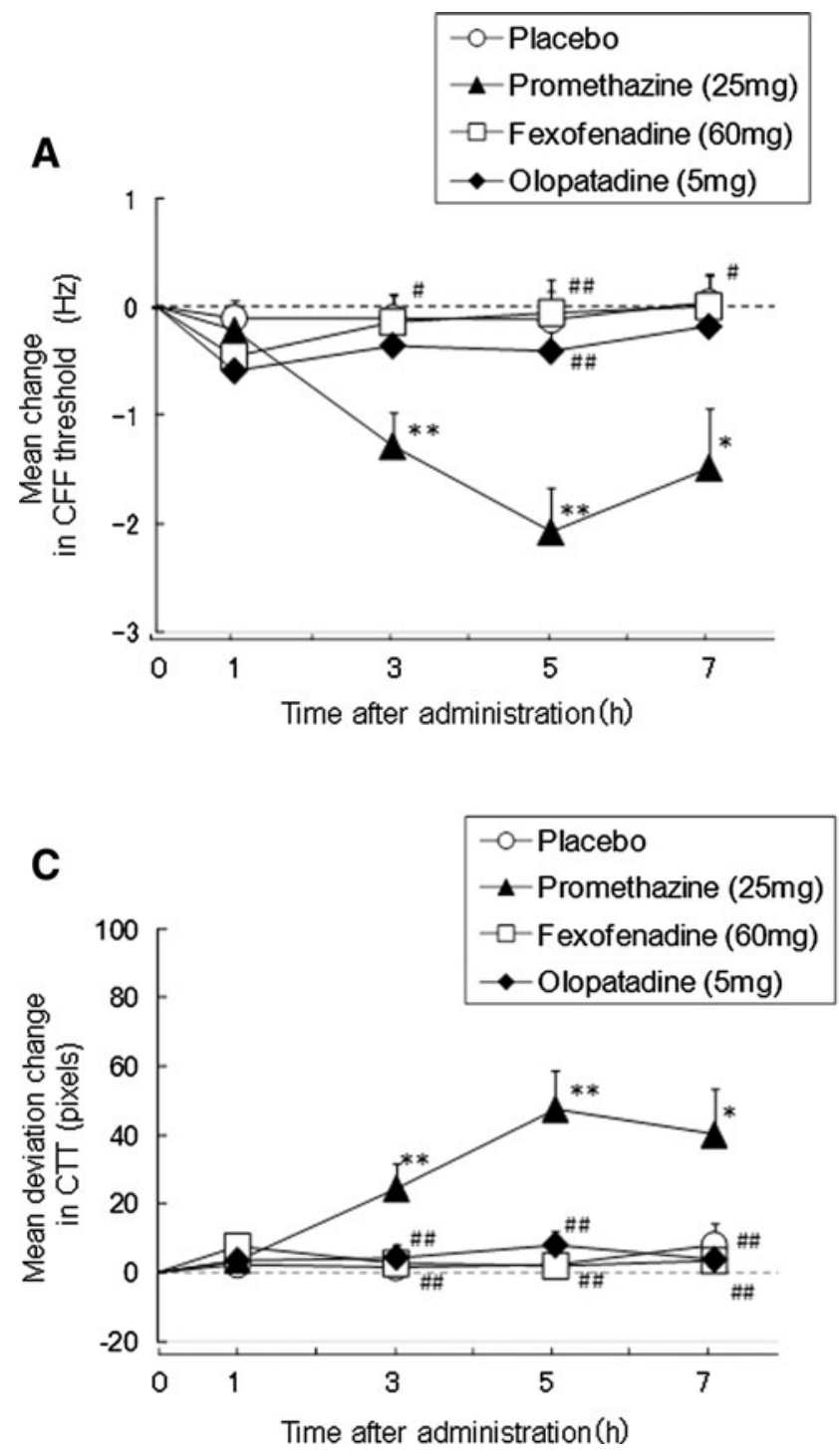

Fig. 3 Effects of antihistamines on CFF (a), CRT (b), tracking ability (c) and recognition reaction time (d) in the CTT. Each value represents the mean change from baseline, and is the mean \pm SEM
$[F(3,276)=1.99, P=0.12]$. There was also significant interaction between treatment and time $[F(9,276)=5.16$, $P<0.01]$. Post hoc pair-wise comparisons indicated that promethazine significantly reduced the CFF thresholds compared with placebo $(P<0.01)$, fexofenadine $(P<0.01)$ and olopatadine $(P<0.01)$. Analysis of the time course showed a significant decrease in CFF thresholds with promethazine in comparison to placebo and fexofenadine, at 3-, 5- and 7-h post-dose. The effects of promethazine were also significantly different from those of olopatadine at $5 \mathrm{~h}$.

For the CRT test (Fig. 3b), a two-factor ANOVA showed a significant main effect of treatment $[F(3,276)=$ $11.11, P<0.01]$, although the effect of time was not significant $[F(3,276)=1.77, P=0.15]$. There was also a significant effect of interaction between treatment and time
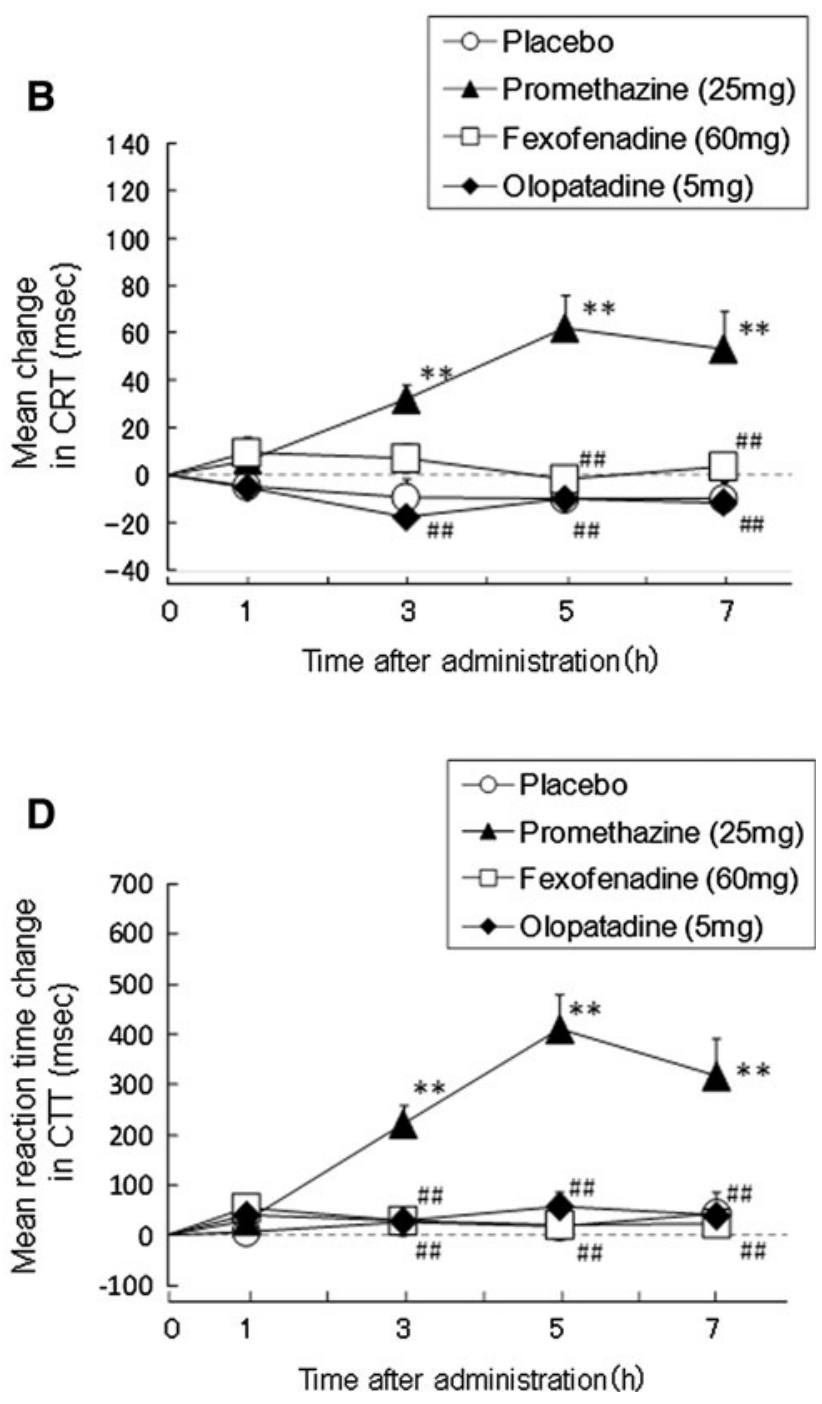

for 24 subjects. $* P<0.05, * * P<0.01$ compared with placebo, ${ }^{\#} P<0.05,{ }^{\# \#} P<0.01$ compared with promethazine 

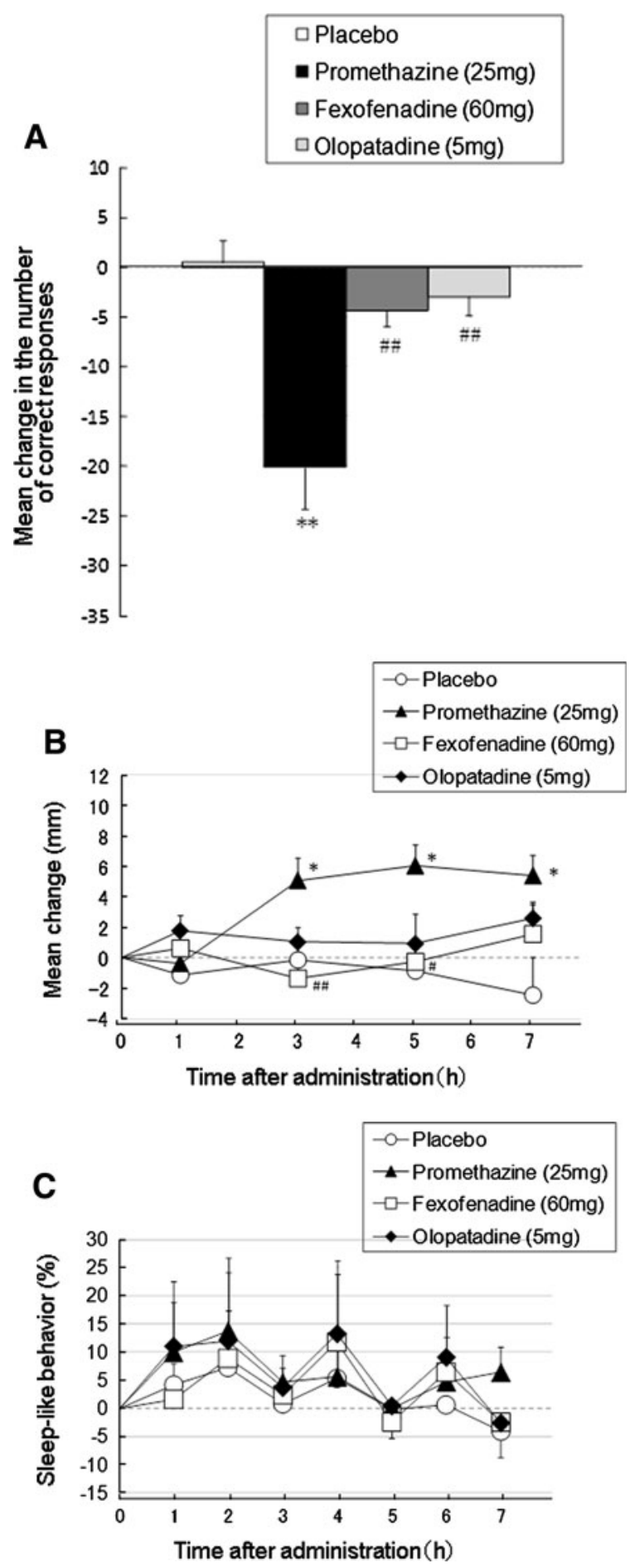

Fig. 4 Effects of antihistamines on RVIP task (a), sedation of LARS (b) and sleep-like activity measured by wrist actigraphy (c). Each value represents the mean change from baseline, and is the mean \pm SEM for 24 subjects. $* P<0.05, * * P<0.01$ compared with placebo, ${ }^{\#} P<0.05,{ }^{\# \#} P<0.01$ compared with promethazine

$[F(9,276)=5.13, P<0.01]$. Post hoc pair-wise comparisons indicated that promethazine significantly increased the response time compared with placebo
$(P<0.01)$, fexofenadine $(P<0.01)$ and olopatadine $(P<0.01)$. Analysis of the time course showed a significant increase in the response time with promethazine in comparison to placebo and olopatadine, at 3-, 5- and 7-h post-dose. The effects of promethazine were also significantly different from those of fexofenadine at 5 and $7 \mathrm{~h}$.

For the tracking task in the CTT test (Fig. 3c), a twofactor ANOVA showed a significant main effect for treatment $[F(3,276)=9.18, P<0.01]$, time $[F(3,276)=$ 6.09, $P<0.01]$ and interaction between treatment and time $[F(9,276)=6.03, P<0.01]$. Post hoc pair-wise comparisons indicated that promethazine significantly impaired tracking ability compared with placebo $(P<0.01)$, fexofenadine $(P<0.01)$ and olopatadine $(P<0.01)$. Analysis of the time course showed a significant impairment in tracking ability with promethazine in comparison to placebo, fexofenadine and olopatadine, at 3-, 5- and 7-h post-dose.

For the peripheral awareness task in the CTT test (Fig. 3d), a two-factor ANOVA showed a significant main effect for treatment $[F(3,276)=14.20, P<0.01]$, time $[F(3,276)=9.52, P<0.01]$ and interaction between treatment and time $[F(9,276)=9.73 ., P<0.01]$. Post hoc pair-wise comparisons indicated that promethazine significantly increased the response time in the periphery of vision compared with the placebo $(P<0.01)$, fexofenadine $(P<0.01)$ and olopatadine $(P<0.01)$. Analysis of the time course showed a significant increase in the response time with promethazine in comparison to placebo, fexofenadine and olopatadine, at 3-, 5- and 7-h post-dose.

For the RVIP test (Fig. 4a), a one-way ANOVA of correct responses showed a significant main effect for treatment $[F(3,92)=12.27, P<0.01]$. Post hoc pairwise comparisons indicated that promethazine significantly decreased the correct responses compared with the placebo $(P<0.01)$, fexofenadine $(P<0.01)$ and olopatadine $(P<0.01)$.

For the sedation score of LARS (Fig. 4b), a two-factor ANOVA showed a significant main effect of treatment $[F(3,276)=4.12, P<0.01]$, although the effect of time was not significant $[F(3,276)=1.51, P=0.21]$. There was also a significant effect of interaction between treatment and time $[F(9,276)=2.82, P<0.01]$. Post hoc pair-wise comparisons indicated that promethazine significantly increased the sedation score compared with placebo $(P<0.05)$. Analysis of the time course showed a significant increase in the sedation score with promethazine in comparison to placebo, at 3-, 5- and 7-h post-dose. The effects of promethazine were also significantly different from fexofenadine at 3- and 5-h post-dose.

A two-factor ANOVA of percentage sleep measured by wrist actigraphy revealed that there was no significant main effect for treatment $[F(3,552)=0.71, P=0.55]$ and the 
effect of interaction between treatment and time $[F(3,552)=$ 1.40, $P=0.13]$. The effect of time was significant $[F(3,552)=12.86, P<0.01]$ (Fig. 4c).

There were no serious adverse events and no subject withdrew due to drug intolerance or any drug-related adverse event.

\section{Discussion}

Second-generation antihistamines are differentiated from the first-generation antihistamines by their increased specificity and efficacy toward histamine $\mathrm{H}_{1}$ receptors, and the low sedative effects due to their much lower penetration into the CNS. However, there are few reports that directly compare the antihistaminic efficacy and impairment of psychomotor functions. The aim of this study was to investigate the effects of fexofenadine and olopatadine on the CNS and their clinical effectiveness by measuring their activity as peripheral inhibitors of histamine-induced wheal and flare. Possible effects on the CNS were assessed using a battery of psychometric tests as shown by previous reports [10, 14].

In the present study, all antihistamines at single therapeutic dosages produced a significant reduction in the wheal and flare responses induced by histamine. Olopatadine showed a rapid and long-lasting inhibitory effect on the histamine-induced cutaneous response. Fexofenadine also had a long-lasting effect. Promethazine, however, had an effect that was less marked and only transient. The results for olopatadine and fexofenadine were consistent with a previous report $[33,34]$. Further, consistent with a previous result [21], the inhibition of histamine-induced itching by olopatadine, but not by fexofenadine, paralleled the inhibitory effect on wheals (data not shown). The rapid effect of olopatadine is due to the fact that its blood concentration reaches a maximum $1 \mathrm{~h}$ after oral administration [5], while the time to reach the maximum blood concentration for promethazine [20] and fexofenadine [33] is 2.7 and $2.2 \mathrm{~h}$, respectively. In addition, the potent and longlasting effect of olopatadine is due to its non-competitive antagonist function to histamine $\mathrm{H}_{1}$ receptors. Namely, the inhibitory effect of olopatadine on histamine receptors is little reduced in the presence of high levels of histamine because of noncompetitive antagonism [16]. These pharmacological and pharmacokinetic characteristics contribute to the clinical effectiveness of olopatadine in patients with seasonal allergic rhinitis [4]. It has been reported that olopatadine at $5 \mathrm{mg}$ caused a marked improvement in nasal discharge and nasal congestion in Japanese patients with cedar pollinosis when they were exposed to cedar pollen in an environmental exposure unit, while fexofenadine at $60 \mathrm{mg}$ did not [4]. These findings suggest that olopatadine is more effective than fexofenadine in improving nasal symptoms of cedar pollinosis.

We also assessed the effects of antihistamines on the CNS. The psychometric tests used in this study have been shown to be valid and reliable measures in the evaluation of cognitive and psychomotor function impaired by sedative antihistamines and other psychoactive substances [9, $10,31]$. The various psychometric tests have been categorized according to their most relevant feature. Namely, Shamsi and Hindmarch [27] have proposed that CFF, CRT, CTT, RVIP, wrist actigraphy and LARS are useful as a means of measuring arousal, psychomotor-speed, sensorimotor co-ordination, attention, and physiological and subjective ratings, respectively.

A single oral administration of promethazine at $25 \mathrm{mg}$, which was used as a positive control, decreased the thresholds of CFF, increased recognition reaction time in the CRT, impaired tracking ability and the peripheral reaction in the CTT, decreased the correct response in the RVIP, and impaired the ratings of LARS used as a subjective assessment of sedation. The results suggest that promethazine produces significant impairments in cognitive and psychomotor function consistent with previous studies [9, 10, 26]. However, promethazine did not affect behavioral activity as measured by wrist actigraphy. Previous report has indicated that promethazine at $30 \mathrm{mg}$ cause a reduction in daytime behavioral activity for up to $6 \mathrm{~h}$ [9]. It is not clear that such discrepancy in the results between wrist actigraphy and other psychomotor function. The wrist actigraphy provides a continuous measurement of behavioral activity, which is different from other psychomotor tests. Since a relaxed environment was offered to subjects when not being tested in waiting room to have them concentrate on other psychomotor tests in this study, the behavioral activity measured by the wrist actigraphy is influenced how subjects spend in the waiting room. It seems that the sedative effects induced by antihistamines was hard to measure by actigraphy, because the space of the waiting room was small, and most of subjects did not often move at there. Thus, the results of wrist actigraphy may be more prone to environmental factors than the other psychomotor tests.

In contrast to promethazine, fexofenadine $(60 \mathrm{mg})$ and olopatadine $(5 \mathrm{mg})$, the second-generation antihistamines, had no significant effect in any test when administered at their therapeutic doses. It has been reported that fexofenadine [17] and olopatadine [28] are selective histamine $H_{1}$ receptor antagonists. On the other hand, promethazine has the potent histamine and acetylcholine receptor antagonisms [1], which are related to the potent sedative effect of promethazine [15]. Fexofenadine did not have any disruptive effects on psychomotor or cognitive performance in healthy European volunteers, when administered at doses of up to $360 \mathrm{mg}$, in a double-blind, placebo-controlled 
study with objective psychometric assessments [10]. On the other hand, we have reported that olopatadine at $10 \mathrm{mg}$, which is double the standard oral dose, caused a decrease in behavioral activity as measured by wrist actigraphy, although it did not affect other psychometric assessments [14]. In this study, olopatadine at $5 \mathrm{mg}$, however, did not cause the change in behavioral activity as measured by wrist actigraphy. The reason for discrepancy between the present result and the previous one may be due to the differences in dose used (single dose vs. double dose, respectively) or environmental factors when not being tested as mentioned above. These findings suggested that fexofenadine and olopatadine at the single therapeutic doses did not cause any cognitive or psychomotor dysfunction.

Recently, second-generation antihistamines have been further classified into two subgroups [12]; those that have no effect at the standard dose, and cause slight sedation at double the standard dose, as seen with cetirizine [38]' and those that do not induce sedation even at exceeded doses, as seen with fexofenadine $[10,38]$. This classification is also supported by studies using PET, in which $0.1 \%$ of cerebral histamine $\mathrm{H}_{1}$ receptors were occupied following treatment with fexofenadine at $120 \mathrm{mg}$ (double the standard oral dose) [38], compared to 12.6 and $25.2 \%$ on treatment with cetirizine at $10 \mathrm{mg}$ (a single dose) and $20 \mathrm{mg}$ (a double dose), respectively [35]. Therefore, the dose-related brain penetration by cetirizine may have contributed to the dose-related cognitive impairment. It has been reported that the cerebral receptor occupancy by olopatadine at $5 \mathrm{mg}$ is $15 \%$ [37], although studies of olopatadine at higher doses have not been done. Thus, olopatadine seems to belong to the same category as cetirizine. The therapeutic dose of olopatadine (single oral dose at $5 \mathrm{mg}$ ) is reasonably safe and suitable in terms of avoiding cognitive and psychomotor impairment, as shown in this study. In fact, olopatadine at $5 \mathrm{mg}$ caused a marked improvement in nasal discharge and nasal congestion in Japanese patients with cedar pollinosis without increasing sleepiness and without decreasing attention [4].

Penetration of the blood-brain barrier is affected by various factors, such as lipophilicity, molecular size and number of hydrogen bonds. Recently, it has been proposed that the limited brain-penetrating capability of secondgeneration antihistamines may be the result of efflux from the CNS via the $p$-glycoprotein pump located at the bloodbrain barrier. Since several second-generation antihistamines including fexofenadine [3] and olopatadine [18] are substrates for $p$-glycoprotein, $p$-glycoprotein-mediated efflux at the blood-brain barrier would result in low brain penetration of these compounds. However, it is unlikely that all second-generation antihistamines have equally low potential to cross the blood-brain barrier because it is possible that some of them modify $p$-glycoprotein efflux activity [19]. Olopatadine, like cetirizine [2], shows a certain amount of penetration, but not fexofenadine.

In conclusion, we found that olopatadine $(5 \mathrm{mg})$ and fexofenadine $(60 \mathrm{mg})$ did not cause any cognitive or psychomotor dysfunction in Japanese volunteers when administered at the therapeutic doses. This result is in contrast to the sedative effect of promethazine $(25 \mathrm{mg})$, a first-generation antihistamine. Further, it is suggested that olopatadine would be a good therapeutic drug with the rapid and longlasting effects for patients with peripheral histamine $\mathrm{H}_{1}$ symptoms.

Acknowledgments This study was supported by a grant from the Nagoya Industrial Science Research Institute and by a 875 Grant for 'Academic Frontier' Project for Private 876 Universities (2007-2011). We thank Miss Azusa Kato, Mr. Tomoya Asahi, Mr. Tomonori Hayakawa and Mr. Hiroshi Yonei for assistance.

Open Access This article is distributed under the terms of the Creative Commons Attribution Noncommercial License which permits any noncommercial use, distribution, and reproduction in any medium, provided the original author(s) and source are credited.

\section{References}

1. Beckmann H, Schmauss M (1983) Clinical investigations into antidepressive mechanisms. I. Antihistaminic and cholinolytic effects: amitriptyline versus promethazine. Arch Psychiatr Nervenkr 233:59-70

2. Chen C, Hanson E, Watson JW, Lee JS (2003) P-glycoprotein limits the brain penetration of nonsedating but not sedating H1-antagonists. Drug Metab Dispos 31:312-318

3. Cvetkovic M, Leake B, Fromm MF, Wilkinson GR, Kim RB (1999) OATP and P-glycoprotein transporters mediate the cellular uptake and excretion of fexofenadine. Drug Metab Dispos 27:866-871

4. Enomoto T, Lu HQ, Yin M, Sakoda T, Dake Y, Enomoto K, Ide T, Cheng L (2009) Evaluation of the efficacy and safety of olopatadine and fexofenadine compared with placebo in Japanese cedar pollinosis using an environmental exposure unit. J Investig Allergol Clin Immunol 19:299-305

5. Fujita K, Magara H, Kobayashi H (1999) Determination of olopatadine, a new antiallergic agent, and its metabolites in human plasma by high-performance liquid chromatography with electrospray ionization tandem mass spectrometry. J Chromatogr B Biomed Sci Appl 731:345-352

6. Hindmarch I, Gudgeon AC (1980) The effects of clobazam and lorazepam on aspects of psychomotor performance and car handling ability. Br J Clin Pharmacol 10:145-150

7. Hindmarch I (1982) Critical flicker fusion frequency (CFF): the effects of psychotropic compounds. Pharmacopsychiatria 15(Suppl 1):44-48

8. Hindmarch I, Shamsi Z (1999) Antihistamines: models to assess sedative properties, assessment of sedation, safety and other sideeffects. Clin Exp Allergy 29:133-142

9. Hindmarch I, Shamsi Z, Stanley N, Fairweather DB (1999) A double-blind, placebo-controlled investigation of the effects of fexofenadine, loratadine and promethazine on cognitive and psychomotor function. Br J Clin Pharmacol 48:200-206 
10. Hindmarch I, Shamsi Z, Kimber S (2002) An evaluation of the effects of high-dose fexofenadine on the central nervous system: a double-blind, placebo-controlled study in healthy volunteers. Clin Exp Allergy 32:133-139

11. Hindmarch I, Subhan Z, Stoker MJ (1983) The effects of zimeldine and amitriptyline on car driving and psychomotor performance. Acta Psychiatr Scand Suppl 308:141-146

12. Holgate ST, Canonica GW, Simons FE (2003) Consensus Group on New-Generation Antihistamines (CONGA): present status and recommendations. Clin Exp Allergy 33:1305-1324

13. Izumi N, Mizuguchi H, Umehara H, Ogino S, Fukui H (2008) Evaluation of efficacy and sedative profiles of $\mathrm{H}(1)$ antihistamines by large-scale surveillance using the visual analogue scale (VAS). Allergol Int 57:257-263

14. Kamei H, Noda Y, Ishikawa Senzaki K, Muraoka I, Hasegawa Y, Hindmarch I, Nabeshima T (2003) Comparative study of acute effects of single doses of fexofenadine, olopatadine, $d$-chlorpheniramine and placebo on psychomotor function in healthy volunteers. Human Psychophamacol 18:611-618

15. Kaneko Y, Shimada K, Saitou K, Sugimoto Y, Kamei C (2000) The mechanism responsible for the drowsiness caused by first generation $\mathrm{H} 1$ antagonists on the EEG pattern. Methods Find Exp Clin Pharmacol 22:163-168

16. Matsumoto Y, Funahashi J, Mori K, Hayashi K, Yano H (2008) The noncompetitive antagonism of histamine $\mathrm{H}_{1}$ receptors expressed in chinese hamster ovary cells by olopatadine hydrochloride: its potency and molecular mechanism. Pharmacology 81:266-274

17. Meeves SG, Appajosyula S (2003) Efficacy and safety profile of fexofenadine $\mathrm{HCl}$ : a unique therapeutic option in $\mathrm{H} 1$-receptor antagonist treatment. J Allergy Clin Immunol 112(Suppl):69-77

18. Mimura N, Nagata Y, Kuwabara T, Kubo N, Fuse E (2008) $P$-glycoprotein limits the brain penetration of olopatadine hydrochloride, H1-receptor antagonist. Drug Metab Pharmacokinet 23:106-114

19. Miura M, Uno T (2010) Clinical pharmacokinetics of fexofenadine enantiomers. Expert Opin Drug Metab Toxicol 6:69-74

20. Moolenaar F, Ensing JG, Bolhuis BG, Jan Visser (1981) Absorption rate and bioavailability of promethazine from rectal and oral dosage forms. Int J Pharm 9:353-357

21. Morita K, Koga T, Moroi Y, Urabe K, Furue M (2002) Rapid effects of olopatadine hydrochloride on the histamine-induced skin responses. J Dermatol 29:709-712

22. Nolen TM (1997) Sedative effects of antihistamine: safety, performance, learning, and quality of life. Clin Ther 19:39-55

23. Parkin C, Kerr JS, Hindmarch I (1997) The effects of practice on choice reaction time and critical flicker fusion threshold. Hum Psychopharmacol 12:65-70

24. Passalacqua G, Scordamaglia A, Ruffoni S, Parodi MN, Canonica GW (1993) Sedation from H1-receptor antagonists: evaluation methods and experimental results. Allergol Immunopathol (Madr) 21:79-83

25. Pechadre JC, Beudin P, Eschalier A, Trolese JF, Rihoux JP (1991) A comparison of central and peripheral effects of cetirizine and loratadine. J Int Med Res 19:289-295

26. Ridoubt F, Hindmarch I (2003) The effects of acute doses of fexofenadine, promethazine, and placebo on cognitive and psychomotor function in healthy Japanese volunteers. Ann Allergy Asthma Immunol 90:404-410

27. Shamsi Z, Hindmarch I (2000) Sedation and antihistamines: a review of inter-drug differences using proportional impairment ratios. Hum Psychopharmacol 15:S3-S30
28. Sharif NA, Xu SX, Miller ST, Gamache DA, Yanni JM (1996) Characterization of the ocular antiallergic and antihistaminic effects of olopatadine (AL-4943A), a novel drug for treating ocular allergic diseases. J Pharmacol Exp Ther 278:1252-1261

29. Simons FE, McMillan JL, Simons KJ (1990) A double-blind, single-dose, crossover comparison of cetirizine, terfenadine, loratadine, astemizole, and chlorpheniramine versus placebo: suppressive effects on histamine-induced wheals and flares during 24 hours in normal subjects. J Allergy Clin Immunol 86:540-547

30. Simons FE (1994) The therapeutic index of newer H1-receptor antagonists. Clin Exp Allergy 24:707-723

31. Stanley N, Hindmarch I (1997) Actigraphy can measure antidepressant induced daytime sedation in healthy volunteers. Hum Psychopharmacol 12:437-443

32. Tagawa M, Kano M, Okamura N, Higuchi M, Matsuda M, Mizuki Y, Arai H, Iwata R, Fujii T, Komemushi S, Ido T, Itoh M, Sasaki H, Watanabe T, Yanai K (2001) Neuroimaging of histamine H1-receptor occupancy in human brain by positron emission tomography (PET): a comparative study of ebastine, a second-generation antihistamine, and ( $\mathrm{t}$ )-chlorpheniramine, a classical antihistamine. Br J Clin Pharmacol 52:501-509

33. Takahashi H, Ishida-Yamamoto A, Iizuka H (2004) Effects of bepotastine, cetirizine, fexofenadine, and olopatadine on histamine-induced wheal-and flare-response, sedation, and psychomotor performance. Clin Exp Dermatol 29:526-532

34. Takahashi H, Zhang Y, Morita E (2008) Evaluation of the antihistamine effects of olopatadine, cetirizine and fexofenadine during a $24 \mathrm{~h}$ period: a double-blind, randomized, crossover, placebo-controlled comparison in skin responses induced by histamine iontophoresis. Arch Dermatol Res 300:291-295

35. Tashiro M, Kato M, Miyake M, Watanuki S, Funaki Y, Ishikawa Y, Iwata R, Yanai K (2009) Dose dependency of brain histamine $\mathrm{H} 1$ receptor occupancy following oral administration of cetirizine hydrochloride measured using PET with [11C]doxepin. Hum Psychopharmacol 24:540-548

36. Tashiro M, Mochizuki H, Iwabuchi K, Sakurada Y, Itoh M, Watanabe T, Yanai K (2002) Roles of histamine in regulation of arousal and cognition: functional neuroimaging of histamine $\mathrm{H} 1$ receptors in human brain. Life Sci 72:409-414

37. Tashiro M, Mochizuki H, Sakurada Y, Ishii K, Oda K, Kimura Y, Sasaki T, Ishiwata K, Yanai K (2006) Brain histamine H receptor occupancy of orally administered antihistamines measured by positron emission tomography with ${ }^{11} \mathrm{C}$-doxepin in a placebocontrolled crossover study design in healthy subjects: a comparison of olopatadine and ketotifen. Br J Clin Pharmacol 61:16-26

38. Tashiro M, Sakurada Y, Iwabuchi K, Mochizuki H, Kato M, Aoki M, Funaki Y, Itoh M, Iwata R, Wong DF, Yanai K (2004) Central effects of fexofenadine and cetirizine: measurement of psychomotor performance, subjective sleepiness, and brain histamine H1-receptor occupancy using 11C-doxepin positron emission tomography. J Clin Pharmacol 44:890-900

39. Van Ruitenbeek P, Vermeeren A, Riedel WJ (2010) Cognitive domains affected by histamine $\mathrm{H}(1)$-antagonism in humans: a literature review. Brain Res Rev 64:263-282

40. Wesnes K, Warburton DM (1983) Effects of smoking on rapid visual information processing performance. Neuropsychobiology 9:223-229

41. Yanai K, Ryu JH, Watanabe T, Iwata R, Ido T, Sawai Y, Ito K, Itoh M (1995) Histamine H1 receptor occupancy in human brains after single oral doses of histamine $\mathrm{H} 1$ antagonists measured by positron emission tomography. Br J Pharmacol 116:1649-1655 